\title{
Asymptotic Analysis of Interference in Cognitive Radio Networks
}

\author{
Yaobin Wen, Sergey Loyka and Abbas Yongacoglu
}

\begin{abstract}
The aggregate interference distribution in cognitive radio networks is studied in a rigorous analytical way using the popular Poisson point process model. While a number of results are available for this model of regular (non-cognitive) networks, cognitive ones present an extra level of difficulties for the analysis, mainly due to the exclusion region around the primary receiver, which are typically addressed via various adhoc approximations (e.g. based on the interference cumulants) or via the large-deviation analysis. Unlike the previous studies, we do not use here ad-hoc approximations but rather obtain the asymptotic interference distribution in a systematic and rigorous way, which also has a guaranteed level of accuracy at the distribution tail. This is in contrast to the large deviation analysis, which provides only the (exponential) order of scaling but not the outage probability itself. Unlike the cumulant-based analysis, our approach provides a guaranteed level of accuracy at the distribution tail. Additionally, our analysis provides a number of novel insights. In particular, we demonstrate that there is a critical transition point below which the outage probability decays only polynomially but above which it decays super-exponentially. This provides a solid analytical foundation to the earlier empirical observations in the literature and also reveals what are the typical ways outage events occur in different regimes. The analysis is further extended to include interference cancelation and fading (from a broad class of distributions). The outage probability is shown to scale down exponentially in the number of canceled nearest interferers in the below-critical region and does not change significantly in the above-critical one. The proposed asymptotic expressions are shown to be accurate in the non-asymptotic regimes.
\end{abstract}

Index Terms-Cognitive radio, wireless network, interference distribution, outage probability, fading, interference cancellation.

\section{INTRODUCTION}

A $\mathrm{S}$ HIGHER data rate services are required in wireless communications over a limited spectrum, there is a need for higher spectrum efficiency. Cognitive Radio (CR), which suggests allowing secondary users (SU) to use the spectrum currently not in use by a primary user (PU), is seen as the main way to overcome the underutilized/overcrowded spectrum problem and to use the available spectrum more efficiently [1]. Due to the uncertainty in the number of SUs and their locations, the PU performance may be seriously

Manuscript received 5 January 2012, revised 16 May 2012, accepted 02 July 2012. This work was presented in part at 25th Biennial Symposium on Communications (QBSC-10), Kingston, Canada, May 2010, at IEEE Vehicular Technology Conference (VTC-10 Fall), Ottawa, Canada, Sep. 2010, and at the IEEE International Conference on Communications, Ottawa, Canada, June 2012.

Y. Wen, S. Loyka and A. Yongacoglu are with the School of Electrical Engineering and Computer Science, University of Ottawa, Ontario, Canada (emails: ywen@rim.com, sergey.loyka@ieee.org, yongacog@site.uottawa.ca).

Digital Object Identifier 10.1109/JSAC.2012.121118. affected by the aggregate interference induced by the SUs in the PU receiver. Therefore, its accurate modeling is important to design cognitive radio networks and also to estimate their potential benefits.

There is an extensive literature dealing with aggregate interference modeling of conventional (non-cognitive) networks [2]. The most popular elements of those models are a Poisson point process (to model the interferers' spatial locations) and the standard propagation path loss models (e.g. path loss exponent and fading). Based on that, Sousa and Silvester [3] studied the aggregate interference power obtaining its characteristic function (CF) and concluding that it is an $\alpha$-stable random variable. Except for some special cases, the closed-form probability density function (PDF) is not available. Using the multivariate Lepage series representation, Ilow and Hatzinakos [4] obtained the $\mathrm{CF}$ of the aggregate interference including the lognormal and Rayleigh fading effects and concluding that the aggregate interference is a spherically symmetric $\alpha$ stable random vector. The benefits of interference cancelation of various forms in wireless networks have been investigated in [5]-[7]. Unlike the previous studies, which were mainly based on the CF approach, [6] studied the tail of the aggregate interference distribution directly and found that, at the low outage regime, the aggregate interference is dominated by the nearest interferer, and this also holds under various fading models and interference cancelation mechanisms. Based on the direct asymptotic analysis, compact and accurate closedform expressions for the outage probability were obtained and a number of insights were pointed out. The beneficial impact of optimum combining using the standard array processing techniques (e.g. MMSE spatial filter) has been studied in [7]. While in some special cases the aggregate interference distribution of a large wireless network approaches the Gaussian one [8], it is far from being accurate in general.

The studies above are limited to the conventional (non-CR) networks. The cognitive ones present additional difficulties for the analysis due to the presence of the forbidden (exclusion) region around the PU receiver where no SUs are allowed to transmit (to ensure the PU's proper quality-of-service), so that the distribution of the aggregate interference is not $\alpha$-stable any more. Consequently, the above-mentioned models/results cannot be used as they are fundamentally based on the $\alpha$-stable nature of the problem. Furthermore, the asymptotic analysis of the interference distribution tail (low outage region) does not apply either, since the forbidden region has a major impact on the tail. These difficulties are addressed in the literature in mainly two typical ways: via various ad-hoc approximations (e.g. using the interference distribution cumulants found from 
its CF) [9][11] or via the large deviation analysis [12]. Additionally, numerical simulations provide a wealth of empirical observations [10]. Since a direct analysis of the interference distribution is challenging while the characteristic function is much more amenable to the analysis, from which its cumulants can be found, this approach was adopted in [9] and [11] to obtain approximations of the interference distribution based on the Edgeworth expansion and truncated stable distributions respectively. While this approach predicts the main body of the distribution well, its accuracy deteriorates significantly at the distribution tail, i.e. at the practically-important low-outage regime (high quality-of-service for the PU). This happens because a limited number of cumulants (typically two) cannot represent well the tail. On the other hand, the use of the large deviation analysis allows one to predict the (exponential) rate of decay of the distribution tail but not the distribution itself (as all constants and slowly-varying functions are neglected in the analysis; in particular, the results are independent of the node density) so that "more refined estimates of the actual probability are needed" [12]. This is accomplished in the present paper.

To overcome the above-mentioned difficulties, we develop a direct approach to the outage probability analysis based on the heavy tail and saddle-point approximation theories. The advantage of this combined approach is that the distribution tail can be found in an explicit closed-form with a guaranteed level of \% accuracy, to the best of our knowledge, for the first time. Additionally, this analysis provides a number of insights and the expressions are also accurate in the nonasymptotic regime (in fact, some of them are accurate over the whole range), and it can also be extended to include the impacts of interference cancelation and fading (from a broad class of distributions), also in combination with each other. Important geometrical and system parameters affecting critically the outage probability are clearly identified.

We have studied a similar CR scenario in [14][15] based on the concept of typical outage events and using ad-hoc approximations in different regimes, which were validated via Monte-Carlo simulations but not via a rigorous analysis. Unlike [14][15], the present paper gives a rigorous analytical evaluation of the asymptotic outage probability (summarized in Theorems 1-4, Corollaries 1-3 and Proposition 4), from which the typical outage events of [14][15] follow as a consequence and do not require any ad-hoc assumptions or approximations.

Using the present approach, we demonstrate that there is a critical point below which the outage probability decays polynomially (i.e. slowly) but above which it decays superexponentially (i.e. very fast), as a function of the threshold interference-to-noise ratio (INR), thus revealing a qualitative transition around this point. This provides a rigorous analytical foundation for the earlier empirical observations in the literature [10], and is also in agreement with a similar phenomenon observed in statistical physics [16]. The analysis also reveals the outage-forming mechanism that is responsible for such behavior: the polynomial (heavy tail) decay is due to a dominant contribution of the nearest active interferer (this also holds under interference cancelation and fading) and the super-exponential decay is due to the aggregate effect of many interferers, none of which is able to cause an outage alone. The main analytical tools are the heavy tail distribution theory for the below-critical region and the saddle-point approximation theory for the above-critical one.

While increasing the forbidden region gives more protection to the PU receiver (by reducing the interference), it also reduces the spectral efficiency of the SUs by allowing fewer of them to transmit. A compromise solution is to decrease the forbidden region and to implement some form of interference cancelation in the PU receiver to keep its quality-of-service at an acceptable level ${ }^{1}$. This configuration is studied in Section IV (to the best of our knowledge, for the first time) by extending the analysis above. The nearest active node is shown to provide the dominant contribution to the outage probability in this case as well, which scales down exponentially in the number of canceled nodes thus demonstrating significant potential of this approach, at the below-critical level. On the contrary, this approach gives little improvement in the abovecritical region. The obtained results are extended in Section $\mathrm{V}$ to include the effect of fading from a broad class of fading distributions whose tails decay faster than polynomially, which includes all popular models, e.g. Rayleigh, Rice, Nakagami, log-normal, Weibull, also in combination with each other and with interference cancelation. In particular, Rayleigh fading is shown to have a negligible impact on the outage probability in the below-critical region and significantly increases it in the above-critical region, while the log-normal fading induces a slight increase in the former and a significantly higher increase in the latter region. Similarly to non-CR networks [2], it is the random CR network geometry that dominates the outage probability in the below-critical region.

The rest of the paper is organized as follows. Section II introduces the system model. In Section III, the rigorous asymptotic analysis of interference is presented, and the nonasymptotic approximations are obtained based on it and validated via Monte-Carlo simulations. The analysis is further extended to the case of interference cancelation in Section IV and fading in Section V Section VI concludes the paper. The proofs are given in the Appendix.

\section{SYSTEM MOdeL}

Let us consider a cognitive radio network which contains a primary user receiver and many secondary users' transmitters (nodes) on a plane. The PU is located at the origin. The SUs are randomly located according to a Poisson point process of the density $\lambda$ [nodes $/ \mathrm{m}^{2}$ ]. Interference coming from the $\mathrm{SU}$ nodes outside the circle of a certain radius $R_{\max }$ is assumed to be negligible (alternatively, no SUs are located outside of this circle, which models a realistic finite-size network). The CR protocol considered here is that all SUs which are inside of a forbidden (exclusion) region (FR), i.e. the circle of the radius $R_{s}$ centered on the $\mathrm{PU}$, cease their transmissions so that some protection to the PU receiver is provided. The interference generated by PUs to each other is assumed to be negligible (which can be achieved by proper frequency planning and/or resource allocation). The geometry of the CR network is illustrated in Fig. 1.

${ }^{1}$ This becomes a feasible solution when the PU is offered some incentives [23] to implement interference cancelation such as a reduced license fee. 


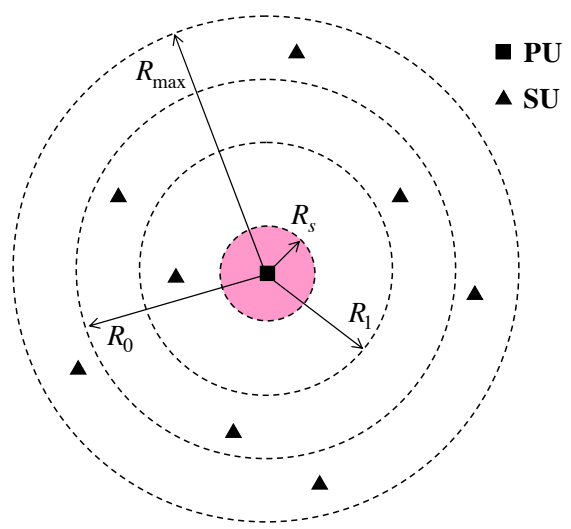

Fig. 1. The geometry of the CR network. Interference coming from the SU nodes outside the circle of the radius $R_{\max }$ is negligible (i.e. the network is finite). The interference coming from the nodes outside of the circle of radius $R_{0}$ is below the noise level at the PU receiver. The average number of nodes in the disk of the radius $R_{1}$ is one. All SUs inside the circle of the radius $R_{s}$ centered on the PU, the forbidden region (FR), cease their transmissions.

We assume that the desired signal, interferences and noise are independent of each other. The total received power at the PU receiver can be expressed as:

$$
P_{P U}=P_{d}+\sum_{i=1}^{N} I_{i}+P_{0}
$$

where $P_{d}$ is the desired signal power; $I_{i}$ is the interference signal power coming from $i$-th SU node; $P_{0}$ is the noise power; $N$ is a Poisson random variable which denotes the number of nodes in the ring between circles of the radii $R_{s}$ and $R_{\max }$, i.e. the potential interference zone where all active interferers are located. The power at the receiver antenna input coming from a transmitter of power $P_{t}$ is given by the standard link budget equation, $P_{r}=P_{t} G_{t} G_{r} g$, where $G_{t}$ and $G_{r}$ are the transmitter and receiver antenna gains; $g$ is the propagation path gain. In this paper, we focus on the impact of the network geometry on the aggregate interference distribution (which is known to have a dominant contribution in regular (non-CR) networks [2]) and consider first a non-fading scenario ${ }^{2}$ so that $g=a_{\nu} r^{-\nu}$ is the average path gain, where $\nu$ is the path loss exponent, $r$ is the distance between the transmitter and receiver, and $a_{\nu}$ is a constant independent of $r$. For simplicity, we assume the transmitter and receiver antennas are isotropic with unity gain, and all SUs transmit at the same constant power level $P_{s}$, so that $P_{r}=P_{s} g$. The $i$-th SU generates the interference power $I_{i}=P_{s} a_{\nu} r_{i}^{-\nu}$ at the PU receiver, where $r_{i}$ is the distance between the $i$-th SU and the PU. Without loss of generality, we normalize $P_{s} a_{\nu}=1$, so that $I_{i}=r_{i}^{-\nu}$. The analysis is further extended to fading channels in Section $\mathrm{V}$, where $g_{i} a_{\nu} r^{-\nu}$ is the propagation path gain and $g_{i}$ is the fading power gain.

Table I summarizes the main notations of this paper.

\footnotetext{
${ }^{2}$ these results will also apply to fading scenarios and delay-insensitive applications, where the PU performance is determined by the average (over fading) powers [10].
}

TABLE I

SUMMARY OF NOTATIONS AND SYMBOLS

\begin{tabular}{|c|c|}
\hline$\lambda$ & the SU node density. \\
\hline$\nu$ & the path loss exponent. \\
\hline$\hat{\theta}$ & a solution of the saddle-point equation. \\
\hline$D$ & $=I_{t h} / P_{0}$, an interference-to-noise (INR) threshold value. \\
\hline$D_{1}$ & the INR from the boundary of the disk with radius $R_{1}$ \\
\hline$D_{\max }$ & $=I_{\max } / P_{0}$, the maximum INR caused by a single node. \\
\hline & $=D / D_{\max }$ \\
\hline$d_{1}$ & $=D / D_{1}$ \\
\hline$I_{a g}$ & the aggregate interference power (at the PU receiver). \\
\hline$I_{1}$ & the nearest node interference power. \\
\hline$I_{t h}$ & the threshold interference power. \\
\hline$I_{k}$ & $k$-th nearest node's interference power. \\
\hline$N_{0}$ & the average number of nodes in the disk of radius $R_{0}$. \\
\hline$N_{\max }$ & the average number of nodes in the disk of radius $R_{\max }$. \\
\hline$N_{r}$ & $\begin{array}{l}=\pi \lambda\left(R_{\max }^{2}-R_{s}^{2}\right) \text {, the average number of nodes } \\
\text { in the annulus of radii } R_{\max } \text { and } R_{s} .\end{array}$ \\
\hline$N_{s}$ & the average number of nodes in the forbidden region. \\
\hline$P_{0}$ & the receiver own noise power. \\
\hline$P_{\text {con }}$ & $\begin{array}{l}\text { the outage probability of the conventional network } \\
\text { (no forbidden region). }\end{array}$ \\
\hline$P_{\text {out }}$ & the outage probability of the CR network. \\
\hline$P_{\text {out }, 1}$ & $\begin{array}{l}=\operatorname{Pr}\left\{I_{1}>I_{t h}\right\}, \text { a probability of the nearest node } \\
\text { interference } I_{1} \text { exceeding the threshold } I_{t h}=D P_{0} .\end{array}$ \\
\hline$P_{\text {out }, k}$ & $\begin{array}{l}=\operatorname{Pr}\left\{I_{k}>I_{t h}\right\}, \text { a probability of } k \text {-th nearest node's } \\
\text { interference } I_{k} \text { exceeding the threshold } I_{t h} \text {. }\end{array}$ \\
\hline$R_{0}$ & a SU at the distance $R_{0}$ from the receiver induces \\
\hline & the average number of nodes in the disk of radius $R_{1}$ is one. \\
\hline$R_{\max }$ & $\begin{array}{l}\text { maximum distance from the } \mathrm{PU} \text { receiver to a SU } \\
\text { (no SU nodes are located outside of this area). }\end{array}$ \\
\hline$R_{s}$ & $\begin{array}{l}\text { the radius of the forbidden region (no active SU nodes } \\
\text { are located inside). }\end{array}$ \\
\hline
\end{tabular}

\section{Aggregate Interference And Outage PROBABILITY}

When the signal to interference plus noise ratio (SINR) is less than a certain threshold $\eta$, there is significant performance degradation of a wireless link and it is considered to be in outage. This is equivalent, under the adopted channel model, to the aggregate interference power $I_{a g}=\sum_{i} I_{i}$ exceeding the threshold $I_{t h}=P_{d} / \eta-P_{0}$, so that the outage probability is

$$
P_{\text {out }}=\operatorname{Pr}\{\operatorname{SINR}<\eta\}=\operatorname{Pr}\left\{I_{a g}>I_{t h}\right\}
$$

We further assume that $P_{d}$ is fixed (e.g. non-fading signal power at the cell boundary). Defining the INR as

$$
\gamma=\sum_{i=1}^{N} I_{i} / P_{0},
$$

its threshold value is $D=I_{t h} / P_{0}$, so that the outage probability can be expressed as

$$
P_{\text {out }}=\operatorname{Pr}\{\gamma>D\}=1-F(D)
$$

where $F(D)$ is the cumulative distribution function (CDF) of the INR. Under the adopted channel model, $P_{\text {out }}$ also serves as a complementary CDF (CCDF) of the aggregate interference $I_{a g}$.

\section{A. Asymptotic Results}

In this section, we present our main contributions in Theorem 1 and 2, and Corollaries 1, 2, which are further extended to the non-asymptotic regime in the next section, and to the 
case of interference cancelation and fading in the following sections.

Since the CR protocol forces all SU nodes inside the forbidden region of the radius $R_{s}$ to cease their transmissions, the interference generated by a single node can not exceed $I_{\max }=R_{s}^{-\nu}$, i.e. the value coming from an active SU node at the closest possible distance, so that the maximum INR caused by a single node is $D_{\max }=I_{\max } / P_{0}$. Let us compare the $\mathrm{CR}$ network with the forbidden region of the radius $R_{s}$ and a corresponding conventional (non-CR) network, which is identical to the $\mathrm{CR}$ one except that there is no forbidden region (i.e. $R_{s}=0$ ). The relationship of their respective outage probabilities $P_{\text {out }}$ and $P_{\text {con }}$ in the asymptotic (low-outage) regime and when $D<D_{\max }$ is characterized as follows.

Theorem 1: Let $d=D / D_{\max }<1$ be the normalized threshold INR bounded away from unity, and $D \rightarrow \infty$. The CR outage probability $P_{\text {out }}$ is then asymptotically proportional to the conventional one $P_{\text {con }}$ (for which $R_{s}=0$ ):

$$
P_{\text {out }}=\operatorname{Pr}\left\{I_{a g}>D P_{0}\right\}=\left(1-d^{2 / \nu}\right) P_{c o n} \cdot(1+o(1))
$$

where $o(\cdot)$ is the small $o$ function ${ }^{3}$.

Proof: see the Appendix.

From Theorem 1 and the corresponding result for the conventional network in [6], it follows that the aggregate interference tail is dominated by the nearest node when $d<1$, which is formalized below.

Corollary 1: Let $P_{\text {out }, 1}=\operatorname{Pr}\left\{I_{1}>I_{t h}\right\}$ be the probability that the nearest node interference exceeds the threshold (thus causing an outage event). The lower bound

$$
P_{\text {out }} \geq P_{\text {out }, 1}
$$

holds for any $D$ and is tight at low $(D \rightarrow 0)$ as well as high INR, when $d<1$ and $D \rightarrow \infty$,

$$
\begin{aligned}
P_{\text {out }} & =P_{\text {out }, 1}(1+o(1)) \\
& =\left(1-d^{2 / \nu}\right) N_{0} D^{-2 / \nu}(1+o(1))
\end{aligned}
$$

where $N_{0}=\pi \lambda R_{0}^{2}$ is the average number of nodes in the disk of radius $R_{0}$.

Proof: see the Appendix.

It follows from Corollary 1 that the lower bound $P_{\text {out }} \geq$ $P_{\text {out }, 1}$ is tight at high INR as well as at low (since $P_{\text {out }, 1} \rightarrow 1$ as $D \rightarrow 0$ ). In fact, this Corollary says that the way a typical (dominant) outage event occurs is when the nearest node interference exceeds the threshold rather than the combined interference from many distant nodes does so. This insight has a dramatic consequence for the interference cancelation considered in Section IV. It is clear from the $(1+o(1))$ term in (5) and (6) that they provide low relative (\%) error and thus can be used as accurate approximations (without $o(1)$ term) for finite but large $D$ (low-outage or high quality-ofservice region) when $D<D_{\max }$, as we demonstrate below in Section III-B. Note that (6) gives the tail behavior of $P_{\text {out }}$ with a guaranteed accuracy level (vanishing $\%$ error as $D \rightarrow \infty$ ) as an explicit function of geometrical and system parameters.

$$
{ }^{3} y=o(x) \text { if } \lim _{D \rightarrow \infty} y / x=0 \text { [19]. }
$$

While Theorem 1 holds for the $D<D_{\max }$ case, the opposite case of $D>D_{\max }$ is also of considerable practical interest for $\mathrm{CR}$ networks. In fact, while the outage probability decreases only polynomially with $D$ in the 1 st case (i.e. comparatively slowly), Corollary 2 below shows that it decreases super-exponentially in the latter case (i.e. very fast), thus revealing a remarkable qualitative transition around the $D=D_{\max }$ point. To understand better why this happens, observe that $d<1$ in Theorem 1 implies $D<D_{\max }$, so that single-node interference can exceed the threshold $D$ and cause an outage event. Corollary 1 further demonstrates that this is a typical outage event. On the other hand, when $D>D_{\max }$, the single-node interference cannot exceed the threshold so that a combined effect of several nodes is required. Let $n=\left\lceil D / D_{\max }\right\rceil$, where $\lceil x\rceil=\min \{n \in \mathbb{Z} \mid n \geq x\}$ is a ceiling function. The outage occurs when the combination of at least $n$ nodes' interference exceeds the threshold. When $D_{\max }<\infty$ and $D \rightarrow \infty$, we have $n \rightarrow \infty$, so that some form of the central limit theorem (CLT) should apply to the combined interference, which results in the Gaussian approximation being a main candidate and an exponential scaling follows. As is well-known, this applies around the mean of the distribution but quickly deteriorates as one moves to the distribution tail, i.e. the region we are interested in (that is the low-outage region), when $n$ is not sufficiently large. To overcome this problem, we use the saddle-point theory (also known as the tilted Edgeworth expansion), which suggest tilting the original distribution in such a way that the point of interest is always around the mean (akin to the importance sampling technique) [13]. An immediate effect is that the relative rather than absolute error becomes small, which has a dramatic positive consequence for the distribution tail [17].

A summary of the saddlepoint approximation theory is given in the following Lemma, which is instrumental for the further analysis.

Lemma 1: Let $Y_{1}, \cdots, Y_{n}$ be independent and identically distributed (i.i.d) random variables with the Moment Generation Function (MGF) $M_{Y}(\theta)=E[\exp (\theta Y)]$, and

$$
\tau_{2}=\sup \left\{\theta: M_{Y}(\theta)<\infty\right\} .
$$

The compound sum $S_{n}=\sum_{i=1}^{n} Y_{i}$, where $n$ is a Poisson random variable with mean $v$, has the following MGF

$$
M_{c}(\theta)=E\left[\exp \left(\theta S_{n}\right)\right]=\exp \left\{v\left(M_{Y}(\theta)-1\right)\right\}
$$

where the subscript $c$ stands for compound. When $M_{Y}(\theta)$ is finite for $\theta<\tau_{2}$ with $\tau_{2}>0$, and $M_{Y}(\theta) \rightarrow \infty$ for $\theta \rightarrow \tau_{2}$, the probability of $S_{n}$ exceeding a threshold $s$ is

$$
\operatorname{Pr}\left(S_{n}>s\right)=Q\left(z_{2}+z_{2}^{-1} \ln \left(z_{3} / z_{2}\right)\right)\left(1+O\left\{\rho_{c 4}(\hat{\theta})\right\}\right)
$$

where

$$
Q(x)=\frac{1}{\sqrt{2 \pi}} \int_{x}^{\infty} \exp \left(-u^{2} / 2\right) d u
$$

is the standard Q-function, $O$ is the big-O function ${ }^{4}, \hat{\theta}$ is a saddle point which is a solution of the stationarity condition

\footnotetext{
${ }^{4} y(x)=O\{g(x)\}$ if $|y(x) / g(x)| \leq C<\infty$ as $x \rightarrow \infty$, where $C$ is a constant [20][19].
} 
$v M_{Y}^{\prime}(\theta)=s$

$$
\begin{aligned}
\rho_{c k}(\hat{\theta}) & =v M_{Y}^{(k)}(\hat{\theta})\left\{v M_{Y}^{\prime \prime}(\hat{\theta})\right\}^{-k / 2} \\
z_{2} & =\operatorname{sgn}(\hat{\theta})\left\{2 \hat{\theta} s-2 v\left(M_{Y}(\hat{\theta})-1\right)\right\}^{1 / 2}, \\
z_{3} & =\hat{\theta}\left\{v M_{Y}^{\prime \prime}(\hat{\theta})\right\}^{1 / 2}
\end{aligned}
$$

and $\operatorname{sgn}(x)=1$ for $x \geq 0$ and -1 otherwise.

Proof: see [[13], Ch.7].

Based on this Lemma, we obtain a rigorous result supporting the intuitive discussion above.

Theorem 2: When $D_{\max }<\infty$ and $D \rightarrow \infty$, which implies $D>D_{\max }$, the CR outage probability can be expressed as:

$$
P_{\text {out }}=Q\left(z_{2}+z_{2}^{-1} \ln \left(z_{3} / z_{2}\right)\right)(1+o(1))
$$

where

$$
\begin{aligned}
z_{2} & =\sqrt{2} \operatorname{sgn}(\hat{\theta})\left(\hat{\theta} d_{1}-J_{-1}-N_{r}\right)^{1 / 2} \\
z_{3} & =\hat{\theta} J_{1}^{1 / 2} \\
J_{k} & =\frac{2}{\nu} \int_{N_{\max }^{-\nu / 2}}^{N_{s}^{-\nu / 2}} \exp (\hat{\theta} x) x^{k-2 / \nu} d x,
\end{aligned}
$$

$d_{1}=D / D_{1}$ is the normalized threshold INR,

$$
D_{1}=1 / R_{1}^{\nu} P_{0}=(\pi \lambda)^{\nu / 2} / P_{0},
$$

$R_{1}$ is the radius of the disk with on average one node in it (so that $D_{1}$ is the INR from the boundary of that $\operatorname{disk}^{5}$ ); $N_{r}=\pi \lambda\left(R_{\max }^{2}-R_{s}^{2}\right)$ is the average number of nodes in the ring between the circles of radii $R_{\max }$ and $R_{s} ; N_{s}=\pi \lambda R_{s}^{2}$ is the average number of nodes in the forbidden region of the radius $R_{s} ; N_{\max }=\pi \lambda R_{\max }^{2}$ is the average number of nodes in the disk of radius $R_{\max }$, and $\hat{\theta}$ is the solution of the following saddle-point equation ${ }^{6}$

$$
d_{1}=\frac{2}{\nu} \int_{N_{\max }^{-\nu / 2}}^{N_{s}^{-\nu / 2}} \exp (\hat{\theta} x) x^{-2 / \nu} d x
$$

An asymptotic analytic solution for the saddle-point $\hat{\theta}$ is

$$
\hat{\theta}=N_{s}^{\nu / 2}(\ln w+\ln \ln w)+o(\ln \ln w)
$$

where $w=\nu N_{s}^{\nu / 2-1} d_{1} / 2$, and

$$
\begin{aligned}
z_{2} & =\sqrt{2} \operatorname{sgn}(\hat{\theta})\left(d_{1}\left(\hat{\theta}-N_{s}^{\nu / 2}\right)-N_{s}\right)^{1 / 2}(1+o(1)) \\
z_{3} & =\hat{\theta}\left(d_{1} N_{s}^{-\nu / 2}\right)^{1 / 2}(1+o(1))
\end{aligned}
$$

Proof: see the Appendix.

Note that Theorem 2 includes two parts: part I is the asymptotic outage probability in (9)-(11) while the saddle-point is evaluated via (12) (e.g. numerically); part II also makes use of (9) but also adds to it the asymptotic expansions in (13)(15). While the Gaussian or Edgeworth expansion [9][8] can provide a small absolute error (and thus non-negligible \% error), the saddle point approximation above gives a small relative $(\%)$ error as shown by the $(1+o(1))$ term in (9),

\footnotetext{
${ }^{5}$ it is also a critical value which separates the high and low outage probability regions in non-CR networks [6].

${ }^{6}$ Unfortunately, this is an integral equation for which an analytic solution is not known. Since it involves a finite-interval integral, it can be solved efficiently using any known numerical technique [22]. Alternatively, (13), (14) and (15) give a closed-form asymptotic solution.
}

so that only the latter is accurate at the distribution tail [17]. The next section shows that (9) can be used as an accurate approximation (without $o(1)$ term) for finite but large $D$ when $D>D_{\max }$, and that the saddlepoint approach reduces the approximation error significantly compared to the Gaussian or Edgeworth approximations. The following Corollary shows that, unlike the outage probability in (6) which decays only polynomially in $D$ (heavy tail), that in (9) decays superexponentially, i.e. much faster [18].

Corollary 2: When $D_{\max }<\infty$ and $D \rightarrow \infty$, the outage probability in (9) decays as

$$
P_{\text {out }}=\exp \left\{-N_{s}^{\nu / 2} d_{1} \ln d_{1}(1+o(1))\right\}
$$

Proof: Combining (13), (14) and (15) with (9) results in (16) after some manipulations.

This qualitative transition in the behavior of $P_{\text {out }}$ around the $D=D_{\max }$ point is in agreement with the earlier empirical observations in the literature [10].

A similar result has been obtained earlier in [12] via the large deviation analysis. In our notations, it can be expressed as (see Theorems 12 in [12]):

$$
P_{\text {out }}=c \cdot \exp \left(-b \cdot d_{1} \ln \left(d_{1}\right)(1+o(1))\right)
$$

where $c$ and $b$ are undetermined constants ${ }^{7}$. Comparing this to (16), one observes that the latter includes the relevant constant and thus can be used to estimate $P_{\text {out }}$, unlike the former. Unfortunately, the $(1+o(1))$ term appears in the exponent and thus creates a significant uncertainty ${ }^{8}$ (see also below). The following Corollary eliminates this uncertainty via an upper bound, leaving vanishing multiplicative uncertainty only.

Corollary 3: When $D_{\max }<\infty$ and $D \rightarrow \infty$, the outage probability in (9) can be upper bounded as

$$
\begin{aligned}
P_{\text {out }} \leq \frac{1}{2} & \exp \left\{-d_{1}\left(a_{1} \ln d_{1}-\frac{N_{\max }^{1+2 / \nu}}{N_{s}}+\frac{N_{r}}{d_{1}}\right)\right\} \\
& \times(1+o(1))
\end{aligned}
$$

where $a_{1}=\frac{1}{2} N_{s}^{\nu / 2}\left(\nu \ln N_{s}-2 \ln \left(2 N_{\max } / \nu\right)\right)$.

Proof: see the Appendix.

Further comparison of (16) and (17) to (9) shows that the latter eliminates any exponential uncertainty and provides low $\%$ error when estimating $P_{\text {out }}$ (provided that a numerical solution is used for (12)), which is also supported by the simulation study below. To understand the implications of the $o(1)$ term better, consider the relative error $\varepsilon$ in (9):

$$
\varepsilon=\frac{P_{\text {out }}-\tilde{P}_{\text {out }}}{P_{\text {out }}}=o(1)
$$

where $\tilde{P}_{\text {out }}$ is the right-hand side of (9) without the $o(1)$ term, i.e. used as an approximation. It follows that $\varepsilon \rightarrow 0$ as $D \rightarrow$ $\infty$, i.e. $\%$ error $\rightarrow 0$. On the other hand, when $\tilde{P}_{\text {out }}$ is the

\footnotetext{
${ }^{7}$ The large deviation analysis does not capture them. In fact, $c$ can also be a function of $d_{1}: c=o\left(d_{1} \ln d_{1}\right)$.

${ }^{8}$ It can be shown that $d_{1}^{p}=e^{o\left(d_{1} \ln d_{1}\right)} \forall p<\infty$ or $d_{1}^{d_{1}^{\alpha}}=$ $e^{o\left(d_{1} \ln d_{1}\right)}, 0<\alpha<1$, i.e. unbounded polynomial or super-polynomial may be present in front of the exponent in (16) and (17).
} 


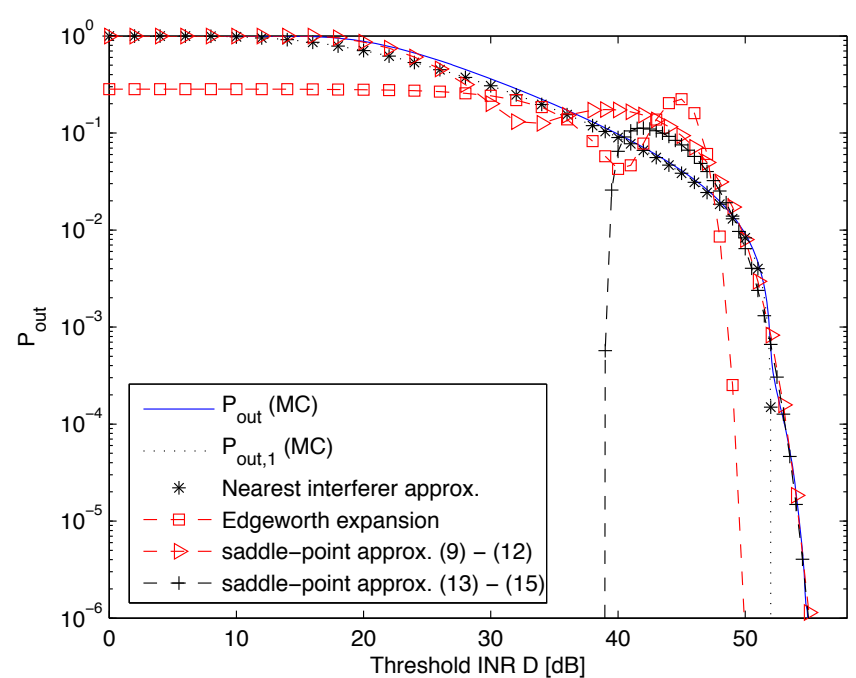

Fig. 2. Outage probability for $D_{\max }>D_{1}\left(R_{s}<R_{1}\right.$, i.e small forbidden region). The simulation parameters are $\nu=4, R_{s}=10 \mathrm{~m}$, $R_{1}=56 \mathrm{~m}, R_{0}=200 \mathrm{~m}, R_{\max }=10^{3} \mathrm{~m}, \lambda=10^{-4}\left[\right.$ nodes $/ \mathrm{m}^{2}$ ], $D_{1}=22 \mathrm{~dB}, D_{\max }=52 \mathrm{~dB}$. MC denotes Monte-Carlo simulations $\left(5 \times 10^{6}\right.$ runs).

right-hand side of (16) or (17) without the $o(1)$ term, one obtains

$$
\varepsilon=e^{o\left(d_{1} \ln d_{1}\right)}
$$

so that $\%$ error may be unbounded $(\rightarrow \infty)$ as the examples in footnote 6 demonstrate. Therefore, the saddle-point asymptotic in Theorem 2 is the best as the $(1+o(1))$ term enters multiplicatively rather than exponentially, providing small \% error.

\section{B. Non-asymptotic outage probability}

The asymptotic results In Theorems 1, 2 and Corollary 1 can be used as approximations (without $o(1)$ terms) for finite but large $D$ under certain conditions, which we summarize below based on the concept of typical outage events by considering 3 typical cases.

- Case 1: $D<D_{\max }$ and $D_{1}<D_{\max }$.

From Theorem 1 and Corollary 1, for finite but large $D$ and $D<D_{\max }, P_{\text {out }} \approx P_{\text {out }, 1}$. The key question is: "When is $D$ sufficiently large so that the approximation is accurate?" From [6], the nearest interferer approximation is accurate in the conventional network when $D>D_{1}$ (i.e. the low-outage regime), where $D_{1}=(\pi \lambda)^{\nu / 2} / P_{0}$ is a critical value which separates the high and low outage probability regions, and is the INR coming from the boundary of the disk (centered on the PU) with on average one node in it. As Theorem 1 links $P_{\text {out }}$ and $P_{\text {con }}$, the corresponding condition also applies to the former, so that large $D$ means $D_{\max }>D>D_{1}$, the aggregate interference is dominated by the nearest node one and $P_{\text {out }} \approx P_{\text {out }, 1}$. This is possible when $D_{1}<D_{\max }$, which implies $R_{s}<R_{1}$, i.e. a small forbidden region. The 1st constraint $D_{1}<D$ can be further removed by observing that $P_{\text {out }, 1} \leq P_{\text {out }} \forall D$ (since one way for an outage to occur is when the nearest node interference exceeds the threshold)

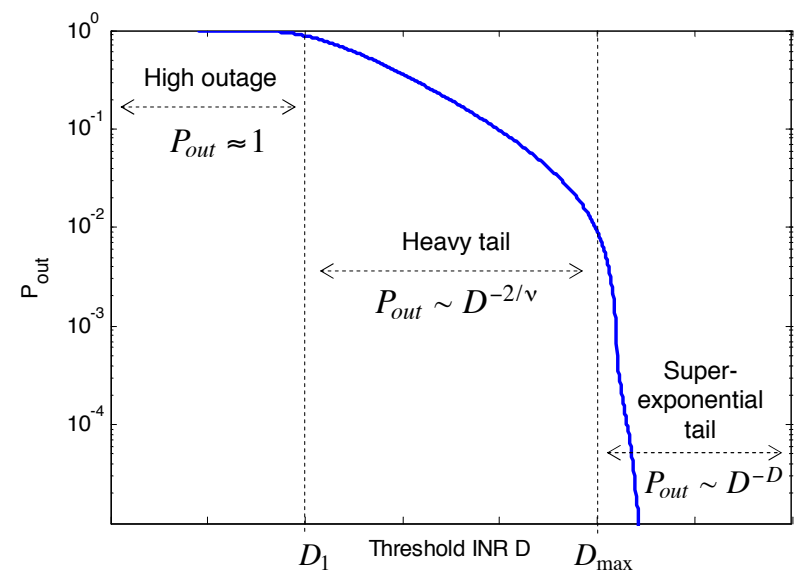

Fig. 3. Three different regimes in the outage probability vs. INR behavior when $D_{1}<D_{\max }$. If $D_{1}>D_{\max }$, the heavy-tail regime disappears.

and that $P_{\text {out }, 1} \rightarrow 1$ as $D \rightarrow 0$ so that $P_{\text {out }, 1} \approx P_{\text {out }}$ in this high-outage regime; finally, $P_{\text {out }} \approx P_{\text {out }, 1} \forall D<D_{\max }$. Fig. 2 shows the outage probability for Case 1 . When $D<D_{\max }$ (heavy tail or below-critical regime), it is well approximated by the nearest node one $P_{\text {out }, 1}$. Note that the standard Edgeworth expansion (e.g. as in [9]) is significantly less accurate in this case. On the other hand, since the nearest node interference cannot exceed $D_{\max }$, the above approximation breaks down when $D>D_{\max }$ (super-exponential or abovecritical regime). These observation have been validated via extensive Monte-Carlo simulations for a wide range of system parameters, of which Fig. 2 is only a sample.

- Case 2: $D>D_{\max }$ and $D_{1}<D_{\max }$.

When $D_{1}<D_{\max }$ (which implies $R_{s}<R_{1}$, i.e. a small forbidden region) and $D>D_{\max }$, a single node contribution cannot cause an outage so that combined contribution of a few nodes is required. On the other hand, the number $n$ of nodes contributing to a typical outage event is not sufficiently large for a practically-important outage range (say $P_{\text {out }}>10^{-10}$ ), so that and Gaussian or Edgeworth approximations are not accurate, as Fig. 2 shows. However, as explained above, the saddlepoint method tilts the original distribution and makes the relative (\%) error small so that its accuracy is much better at the tail. Fig. 2 shows the saddlepoint approximation for $R_{s}<$ $R_{1}$. When $D>D_{\max }$, its accuracy is remarkably good. On the other hand, when $D<D_{\max }$, the saddlepoint approximation is not accurate since the aggregate interference is dominated by the nearest node one and the CLT-type argument is not expected to work well in this case, so that Theorems 1 and 2 are essentially complementary to each other, providing a combined accurate approximation of $P_{\text {out }}$ for the whole INR range via three typical regimes, which is summarized below:

$\diamond$ High-outage regime: $D<D_{1}$ so that $P_{\text {out }} \approx 1$. Nearest node dominates.

$\diamond$ Below-critical (heavy tail) regime: $D_{1}<D<D_{\max }$ so that $P_{\text {out }} \approx P_{\text {out }, 1} \approx\left(1-d^{2 / \nu}\right) N_{0} D^{-2 / \nu}$. Nearest node dominates.

$\diamond$ Above-critical (super-exponential tail) regime: $D>D_{\max }$ so that $P_{\text {out }}$ is as in (9) and (16). Several nodes contribute to typical outage events. 


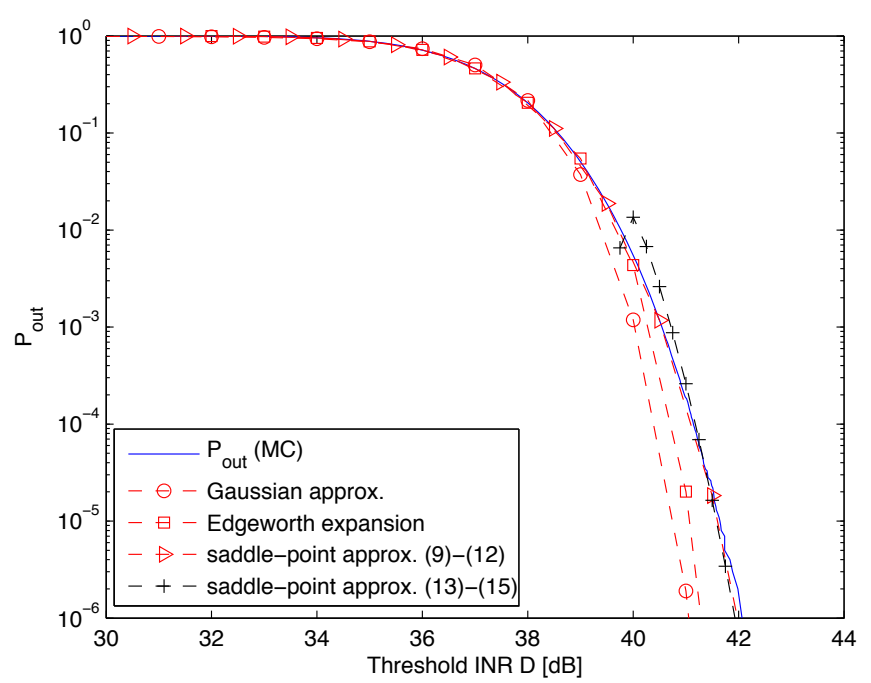

Fig. 4. Outage probability for $D_{1}>D_{\max }\left(R_{s}>R_{1}\right.$, i.e. large forbidden region). The simulation parameters are $\nu=4, R_{s}=32 \mathrm{~m}, R_{1}=18 \mathrm{~m}$, $R_{0}=200 \mathrm{~m}, R_{\max }=10^{3} \mathrm{~m}, \lambda=10^{-3}\left[\right.$ nodes $\left./ \mathrm{m}^{2}\right], D_{1}=42 \mathrm{~dB}, D_{\max }=$ $32 \mathrm{~dB}$. MC denotes Monte-Carlo simulations ( $10^{6}$ runs).

These regimes are illustrated in Fig. 3. It is interesting to observe that these regimes are also inline with the corresponding observations in [16] which identify different modes of convergence for truncated Levy flights.

- Case 3: $D_{1}>D_{\max }$.

When $D_{1}>D_{\max }$, which implies $R_{s}>R_{1}$, i.e. a large forbidden region, $n$ is large in the low outage range, i.e. a typical outage event is when the combination of interference from many nodes exceeds the threshold, and the aggregate interference is closely approximated by a Gaussian random variable, $\sum_{i=1}^{N} I_{i} \sim \mathcal{N}$ [10][15]. As Fig. 4 demonstrates, the Gaussian and Edgeworth approximations are accurate with 1$2 \mathrm{~dB}$ gap at the tail, and the saddlepoint approximation is the most accurate one with $0.1 \mathrm{~dB}$ gap at the tail. The closed-form expression of the saddlepoint approximation is less accurate than the numerical one, since the former was based on the asymptotic expansions in (13) - (15).

\section{IMPACT OF INTERFERENCE CANCELATION}

In a CR network and a location-based protocol, some form of protection to the PU receiver is provided by not allowing all the SUs in the forbidden region (FR) to transmit. Clearly, the larger the FR, the smaller the interference generated by the remaining (active) SUs, but also the lower the SU spectral efficiency as fewer SUs are allowed to transmit. A compromise solution is to reduce the FR (so that more SUs are allowed to transmit) and to implement an interference cancelation scheme $^{9}$ in the PU receiver (so that its quality of service is maintained). In this section, we analyze this scenario using the asymptotic tools developed above.

Theorem 3: Consider the scenario where, in an addition to the forbidden region, $(k-1)$ nearest (to the PU receiver) interferers located outside the FR are canceled. When $d=$

${ }^{9}$ e.g. null forming by an antenna array as in [24] or any other suitable technique.
$D / D_{\max }<1$ and $D \rightarrow \infty$, the CR outage probability $P_{\text {out }}=$ $\operatorname{Pr}\left\{\sum_{i=k}^{N} I_{i}>D P_{0}\right\}$ is still dominated by the nearest active $(k$-th) node and can be expressed as:

$$
\begin{aligned}
P_{\text {out }} & =P_{\text {out }, k}(1+o(1))=\frac{1}{k !} P_{\text {out }, 1}^{k}(1+o(1)) \\
& =\frac{1}{k !}\left(\frac{N_{0}}{D^{2 / \nu}}\left(1-d^{2 / \nu}\right)\right)^{k}(1+o(1))
\end{aligned}
$$

where $P_{\text {out }, k}=\operatorname{Pr}\left\{I_{k}>D P_{0}\right\}$ is the probability that $k$-th nearest node's interferer exceeds the threshold, and $P_{\text {out }, 1}$ is that without interference cancelation $(k=1)$. The lower bound

$$
P_{\text {out }} \geq P_{\text {out }, k}
$$

holds for any $D$ and is tight at high $(D \rightarrow \infty)$ as well as low $(D \rightarrow 0)$ INR.

Proof: see the Appendix.

Note from (21) that $P_{\text {out }}$ scales down faster than exponentially in $k$, so that canceling nearest active interferers pays off well, allowing to maintain low $P_{\text {out }}$ for the PU receiver and to increase the spectral efficiency of the SUs. To see this effect in more details, consider the ratio:

$$
\frac{P_{\text {out },(k+1)}}{P_{\text {out }, k}}=\frac{N_{0}\left(1-d^{2 / \nu}\right)}{D^{2 / \nu}(k+1)}(1+o(1))
$$

Clearly, canceling an extra nearest interferer has a significant beneficial effect for large $D$. Compared to the conventional network $(d=0)$, the impact of the forbidden region on the outage probability is captured by the $\left(1-d^{2 / \nu}\right)^{k}$ term in (21).

Corollary 1 can now seen as a special case of Theorem 3 with $k=1$. Similarly to the no interference cancelation case $(k=1),(21)$ can serve as an approximation (without $o(1)$ term) when $D<D_{\max }$,

$$
P_{\text {out }} \approx P_{\text {out }, k} \approx \min \left\{1,\left(N_{0} D^{-2 / \nu}\left(1-d^{2 / \nu}\right)\right)^{k} / k !\right\}
$$

where we have used the fact that $1 \geq P_{\text {out }} \geq P_{\text {out }, k}$, i.e. that $P_{\text {out }}$ is at least as large as that due to $k$-th nearest node and cannot exceed one. To validate this approximation, Fig. 5 shows the outage probability under interference cancelation when $R_{s}<R_{1}$ (small forbidden region) ${ }^{10}$. Note that the approximation in (22) is quite accurate when $D<D_{\max }$. It is also clear that canceling nearest active node reduces significantly $P_{\text {out }}$ in this below-critical regime. For the nonfading $k=1$ case, also note the transition from the heavy tail region (polynomial decrease in $P_{\text {out }}$ as in (22)) to the steep decrease region (super-exponential, as in (18)) around the $D=D_{\max }=52 \mathrm{~dB}$ point.

\section{IMPACT OF FADING}

In this section, we study the impact of fading on the aggregate interference distribution. Let us consider the ordered average interference powers $I_{1} \geq I_{2} \geq \cdots \geq I_{N}$ which are further subjected to fading so that the fading received powers are $I_{g i}=g_{i} I_{i}$, where $g_{i}$ are the fading power gains, assumed to be i.i.d. with the PDF $f_{g}(x)$ and the $\operatorname{CDF} F_{g}(x)^{11}$.

\footnotetext{
${ }^{10}$ Since $D_{\max }=52 \mathrm{~dB}$ is a constant in this figure, $d=D / D_{\max }$ becomes a variable along with $D$.

${ }^{11}$ Here we tacitly assume that the required signal is not fading, e.g. due to line-of-sight propagation. Alternatively, our results represent the CCDF of the aggregate interference.
} 


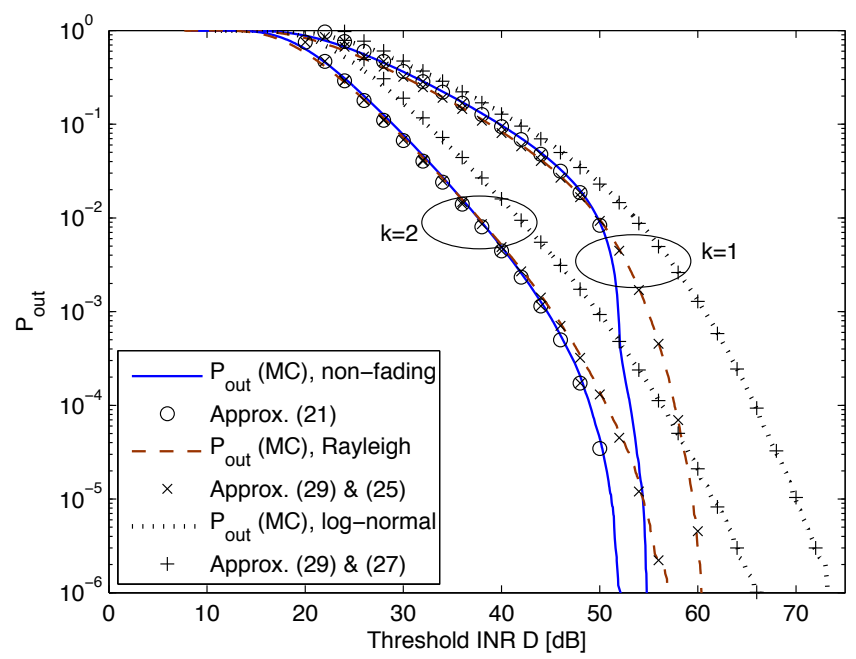

Fig. 5. Outage probability with interference cancelation and fading when $R_{s}<R_{1}$ (small forbidden region). The simulation parameters are $\nu=4$, $R_{s}=10 \mathrm{~m}, R_{1}=56 \mathrm{~m}, R_{0}=200 \mathrm{~m}, R_{\max }=10^{3} \mathrm{~m}, \lambda=$ $10^{-4}\left[\right.$ nodes $\left./ \mathrm{m}^{2}\right], D_{1}=22 \mathrm{~dB}, D_{\max }=52 \mathrm{~dB} ; \sigma=6 \mathrm{~dB}$. MC denotes Monte-Carlo simulations $\left(5 \times 10^{6}\right.$ runs $)$.

\section{A. Asymptotic Results}

The theorem and corollary below characterize the asymptotic outage probability under fading (from a broad class of distributions) alone and both fading and interference cancelation simultaneously.

Theorem 4: Let $d=D / D_{\max }<1$ and the fading distribution tail decays faster than polynomially, i.e.

$$
\lim _{x \rightarrow \infty}\left(1-F_{g}(x)\right) x^{p}=0 \forall p<\infty,
$$

where $F_{g}(x)$ is the $\mathrm{CDF}$ of the fading factor. When $D \rightarrow \infty$, the outage probability $P_{\text {out }}=\operatorname{Pr}\left\{\sum_{i} I_{g i}>D P_{0}\right\}$ is dominated by the nearest node interference:

$$
\begin{aligned}
P_{\text {out }} & =\operatorname{Pr}\left\{I_{g 1}>D P_{0}\right\}(1+o(1)) \\
& =N_{0} D^{-2 / \nu} M_{1}(d) \cdot(1+o(1))
\end{aligned}
$$

where

$$
M_{k}(d)=\int_{d}^{\infty}\left(g^{2 / \nu}-d^{2 / \nu}\right)^{k} f_{g}(g) d g
$$

is a biased moment of $g$ of order $2 k / \nu$ (when $d=0$, it is the moment of $g$ of order $2 k / \nu$ ).

Proof: see the Appendix.

Note that $M_{1}(d)$ includes the impact of both the forbidden region and fading. The impact of fading alone is quantified, via comparison of (6) and (23), by $M_{1}(d)\left(1-d^{2 / \nu}\right)^{-1}$ : if the latter is greater than 1 , the impact is negative (higher $P_{\text {out }}$ due to fading), and positive otherwise.

Based on Theorem 4, the impact of fading and interference cancelation can now be quantified.

Proposition 4: Under the conditions of Theorem 4 and when $(k-1)$ nearest nodes are canceled, the outage probability is dominated by the nearest active ( $k$-th) node:

$$
\begin{aligned}
P_{\text {out }} & =\operatorname{Pr}\left\{g_{k} I_{k}>D P_{0}\right\}(1+o(1)) \\
& =\frac{1}{k !}\left(\frac{N_{0}}{D^{2 / \nu}}\right)^{k} M_{k}(d)(1+o(1)) .
\end{aligned}
$$

\section{Proof: see the Appendix.}

Note that $\left(N_{0} D^{-2 / \nu}\right)^{k} / k$ ! represent the conventional outage probability, i.e. no forbidden region and fading, and $M_{k}(d)$ quantifies the impact of the latter two.

It should be emphasized that Theorem 4 and Proposition 4 allow for a broad class of fading distributions, encompassing all popular models such as Rayleigh, Rice, Nakagami, Weibull and log-normal (also in combination with each other), whose tail decays faster than polynomially.

Theorem 4 can be considered as a generalization of Theorem 1: the latter can be recovered from the former using $f_{g}(x)=\delta(x-1)$. It can also be considered as a generalization of Theorem 4 in [6]: the latter can be recovered by using $d=0$, i.e. no forbidden region.

Let us now specify the results above to the two popular fading models.

Rayleigh fading (small scale): The power gain PDF is $f_{g}(x)=\exp (-x)$. The outage probability is as in Theorem 4 and Proposition 4 with,

$$
M_{k}(d)=\sum_{i=0}^{k} C_{k}^{i}(-1)^{i} d^{2 i / \nu} \Gamma(2(k-i) / \nu+1, d)
$$

where $C_{k}^{i}=k ! /(i !(k-i) !)$ is the binomial coefficient and $\Gamma(a, x)=\int_{x}^{\infty} t^{a-1} e^{-t} d t$ is the incomplete Gamma function. In particular, when $k=1$, i.e. no interference cancelation,

$$
M_{1}(d)=\Gamma(2 / \nu+1, d)-d^{2 / \nu} e^{-d}
$$

Log-normal fading (large scale): The power gain PDF is

$$
f_{g l}(x)=\frac{1}{\sqrt{2 \pi} x \sigma} \exp \left(-\frac{\ln ^{2} x}{2 \sigma^{2}}\right),
$$

where $\sigma$ is the standard deviation of $\ln x$ in natural units, and the impact of fading and forbidden region is given by

$$
\begin{aligned}
M_{k}(d)= & \sum_{i=0}^{k}(-1)^{i} d^{2 i / \nu} C_{k}^{i} \exp \left(\frac{2 \sigma^{2}(k-i)^{2}}{\nu^{2}}\right) \\
& \times Q\left(\frac{\ln d}{\sigma}-\frac{2 \sigma(k-i)}{\nu}\right)
\end{aligned}
$$

When $k=1$, i.e. no interference cancelation,

$$
M_{1}(d)=\exp \left(\frac{2 \sigma^{2}}{\nu^{2}}\right) Q\left(\frac{\ln d}{\sigma}-\frac{2 \sigma}{\nu}\right)-d^{2 / \nu} Q\left(\frac{\ln d}{\sigma}\right)
$$

\section{B. Non-asymptotic outage probability}

Similarly to the non-fading scenario, the asymptotic results above can be used as approximations (without $o(1)$ terms) for finite but large $D$ under certain conditions, which we summarize below based on the concept of typical outage events by considering 3 typical cases depending on the size of the forbidden region.

- Case 1: $R_{s}<R_{1}$ (small forbidden region). 


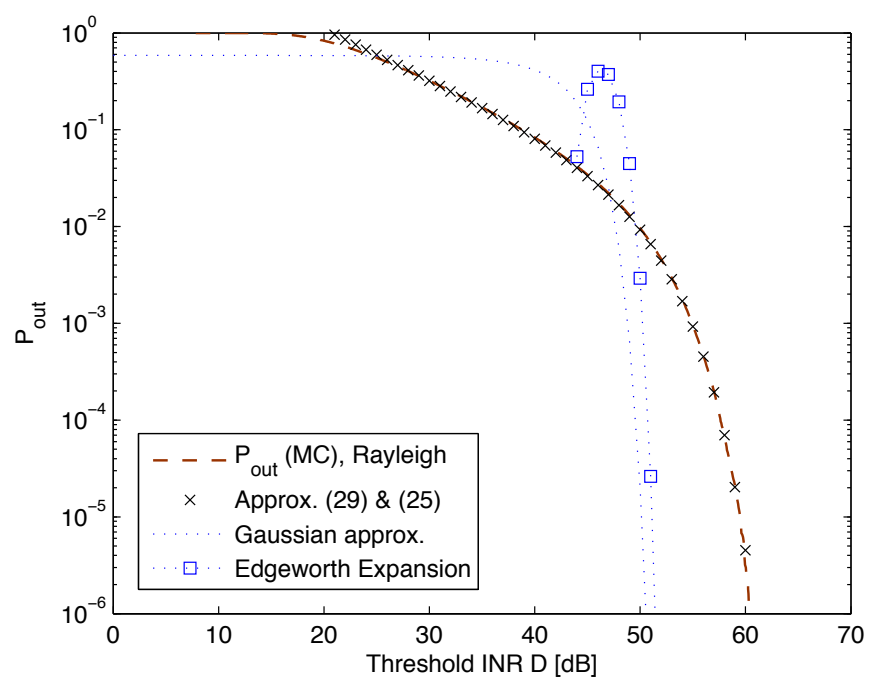

Fig. 6. Outage probability under Rayleigh fading as in Fig. $5(k=1)$ : comparison to Gaussian and Edgeworth expansions.

From Theorem 4 and Proposition 4, for finite but large $D<$ $D_{\max }$, the outage probability is dominated by the $k$-th nearest node after the cancelation, and thus can be approximated by (24) without the $o(1)$ term,

$$
P_{\text {out }} \approx \frac{1}{k !}\left(\frac{N_{0}}{D^{2 / \nu}}\right)^{k} M_{k}(d)
$$

Similar to the non-fading case, this approximation holds when $D_{1}<D<D_{\max }$, which implies $D_{1}<D_{\max }$, which is equivalent to $R_{s}<R_{1}$, i.e. a small forbidden region. The condition $D_{1}<D$ can be relaxed by noting that $P_{\text {out }, k} \leq$ $P_{\text {out }} \leq 1$, i.e. via the combined approximation/lower bound,

$$
P_{\text {out }} \approx \min \left\{1, \frac{M_{k}(d)}{k !}\left(\frac{N_{0}}{D^{2 / \nu}}\right)^{k}\right\}
$$

To evaluate the approximation accuracy, Fig. 5 illustrates this case under Rayleigh and log-normal fading distributions. As expected, when $D<D_{\max }, P_{\text {out }}$ is well approximated by the contribution of the nearest active node as in (29), which is a lower bound in the general case.

Extensive simulation experiments for a wide range of system parameters (of which Fig. 5 is only a sample) show that the above approximation holds even when $D>D_{\max }$. We attribute this to the fact that when fading is present, the nearest node contribution can exceed $D_{\max }$, and the outage probability is dominated by the nearest node in a positive fading state $(g>1)$. Comparing log-normal and Rayleigh fading in this case, both have a minor impact when $D<D_{\max }$ (with the impact of Rayleigh fading hardly noticeable at all) and both have a major impact when $D>D_{\max }$, with the former far outweighing the latter, which is explained by the longer tail of the $\log$-normal distribution $\left(\exp \left(-(\ln x)^{2}\right)\right.$ vs. $\exp (-x))$. Thus, similarly to non-CR networks [2], it is the random network geometry that dominates the outage probability when $D<D_{\max }$. As Fig. 6 shows, the nearest neighbor approximation in (29), (30) is much more accurate than the Gaussian or Edgeworth expansions over the whole INR range

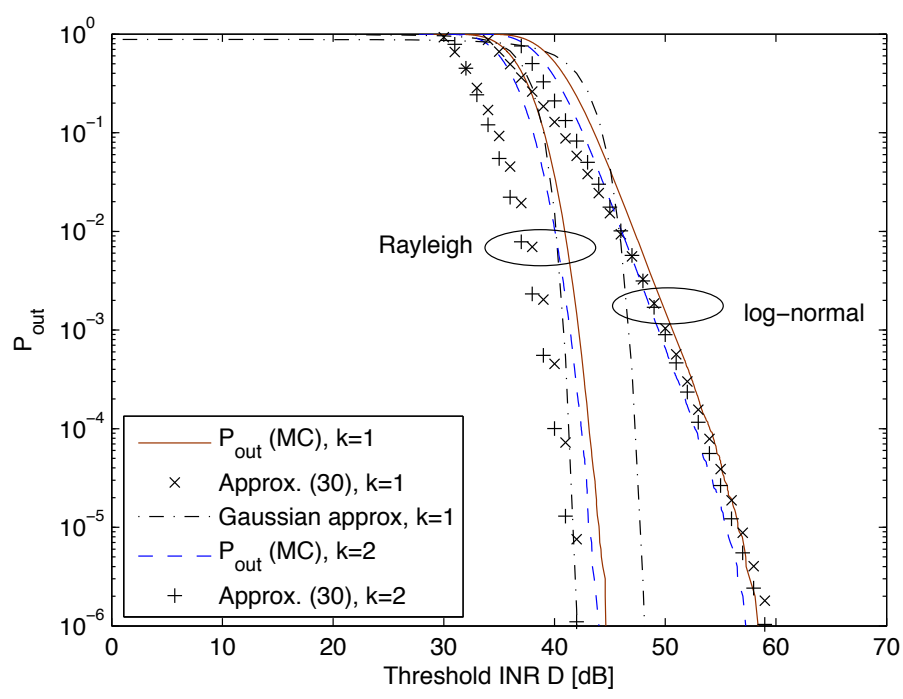

Fig. 7. Outage probability with interference cancelation and fading when $R_{s}>R_{1}\left(D_{1}>D_{\max }\right)$. The simulation parameters are $\nu=4, R_{s}=32 \mathrm{~m}$, $R_{1}=18 \mathrm{~m}, R_{0}=200 \mathrm{~m}, R_{\max }=10^{3} \mathrm{~m}, \lambda=10^{-3}\left[\right.$ nodes $\left./ \mathrm{m}^{2}\right], D_{1}=$ $42 \mathrm{~dB}, D_{\max }=32 \mathrm{~dB} ; \sigma=6 \mathrm{~dB}$. MC denotes Monte-Carlo simulations (10 6 runs).

and it is also able to predict accurately the outage probability in all three distinct regions (high outage $(D<20 \mathrm{~dB})$, heavy (polynomial) tail $(20<D<50 \mathrm{~dB})$ and exponential $(D>50$ $\mathrm{dB})$ ) and this also holds for log-normal fading.

- Case 2: $R_{s}>R_{1}$ (large forbidden region).

Because of the effect of fading, single node INR can exceed $D_{\max }$ when it is in a positive fading state $(g>1)$, so that single node can still cause an outage even when $D>D_{\max }$. The typical outage event is when the combined interference from a few nearest nodes in positive fading states exceed the threshold. As Fig. 7 shows, the approximation in (30) is still reasonably accurate, especially for the log-normal fading ${ }^{12}$. We attribute this to the fact that, while many nodes contribute to the typical outage event in the high-outage region (before the steep transition region) so that the Gaussian approximation is appropriate, it is a few nearest nodes plus positive fading that is dominant in the steep transition low-outage region, where the Gaussian approximation is remarkably less accurate. Also note that interference cancelation does not bring significant advantage in this case: since there is no dominant node, canceling the nearest one does not help much. As far as the approximation in (29) is concerned, the $M_{k}(d)$ term provides a dominant contribution in this case, which explains why $P_{\text {out }}$ is not reduced significantly by going from $k=1$ to $k=2$.

\section{CONCLUSION}

This paper has provided the accurate, closed-form outage provability approximations for CR networks, which are based on the asymptotic analysis of interference and the saddlepoint theory. They can serve as an important evaluation tool in the deployment of the future CR networks since they provide insights into the interference-generating mechanism

${ }^{12}$ Since $D_{\max }=32 \mathrm{~dB}$ is a constant in this figure, $d=D / D \max$ becomes a variable along with $D$. 


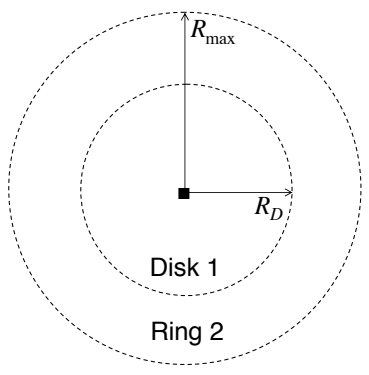

(a) conventional network

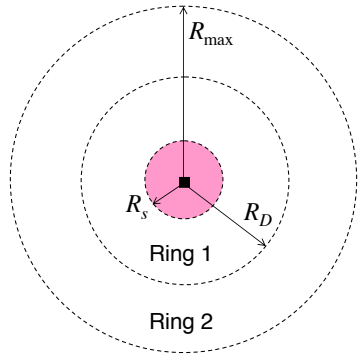

(b) cognitive radio network
Fig. 8. Geometry of conventional (no forbidden region) and CR wireless networks. Disk 1 and Ring 1 are active interference zones (presence of a single node there causes an outage event), while Ring 2 is a potential interference zone (several nodes there are required to cause an outage event).

and suggest ways to combat it, and include the system and network parameters in a clear way. Unlike the prior results, these expressions are accurate in the low-outage region. The asymptotic analysis revealed the qualitative transition in the outage probability behavior around the critical point, when the decay changes from polynomial to super-exponential, thus providing a rigorous analytical foundation for the earlier empirical observations in the literature. Typical outage events have been identified, which farther facilitate a reduction in the outage probability via e.g. interference cancelation. The impacts of fading and interference cancelation, either alone or in combination with each other, have been also included in the analysis. In particular, the outage probability is shown to scale down exponentially in the number of canceled nearest interferers in the below-critical region. All results and conclusions are validated via extensive Monte-Carlo simulations.

\section{APPENDIX}

\section{A. Proof of Theorem 1}

Let $n_{D}$ be the number of nodes in disk 1 of the radius $R_{D}$ in Fig. 8.a, and $n_{1}$ be the number of nodes in ring 1 which is between the circles of radii $R_{s}$ and $R_{D}$ in Fig. 8.b. Let ring 2 be the ring between the circles of radii $R_{D}$ and $R_{\max }$ in Fig. 8. Define the following probabilities:

$$
\begin{aligned}
& P_{1}=\operatorname{Pr}\left\{n_{D} \geq 1\right\}, P_{3}=\operatorname{Pr}\left\{n_{1} \geq 1\right\} \\
& P_{2}=\operatorname{Pr}\left\{\sum_{i \subseteq \text { ring } 2} I_{i}>D P_{0}\right\}
\end{aligned}
$$

The conventional outage probability can be now expressed as:

$$
P_{\text {con }}=P_{1}+\left(1-P_{1}\right) P_{2}
$$

From the Poisson point process, $P_{1}=1-\exp \left(-\lambda \pi R_{D}^{2}\right)$, and from Theorem 1 in [6], as $D \rightarrow \infty$,

$$
\left(1-P_{1}\right) P_{2} / P_{1}=o(1)
$$

The CR outage probability can be expressed as:

$$
P_{\text {out }}=P_{3}+\left(1-P_{3}\right) P_{2}
$$

Since $D=d \cdot D_{\max }$, one obtains

$$
P_{3}=1-\exp \left(-\lambda \pi R_{D}^{2}\left(1-d^{2 / \nu}\right)\right) .
$$

When $d<1$ and $D \rightarrow \infty$, the limit of the ratio of $P_{\text {out }}$ and $P_{\text {con }}$ can be evaluated as follows:

$$
\begin{aligned}
\lim _{D \rightarrow \infty} \frac{P_{\text {out }}}{P_{\text {con }}}= & \lim _{D \rightarrow \infty}\left(\frac{P_{3}}{P_{1}}+\frac{\left(1-P_{3}\right) P_{2}\left(1-P_{1}\right)}{P_{1}\left(1-P_{1}\right)}\right) \\
& \times\left(1+\frac{\left(1-P_{1}\right) P_{2}}{P_{1}}\right)^{-1} \\
= & \left(1-d^{2 / \nu}\right)
\end{aligned}
$$

from which (5) follows.

\section{B. Proof of Corollary 1}

From [[6] Theorem 1], when $D \rightarrow \infty$,

$$
P_{\text {con }}=N_{0} D^{-2 / \nu} \cdot(1+o(1))
$$

where $N_{0}=\pi \lambda R_{0}^{2}$. When $d<1$ and $D \rightarrow \infty$, one obtains from (5) and (33),

$$
P_{\text {out }}=N_{0}\left(D^{-2 / \nu}-D_{\max }^{-2 / \nu}\right) \cdot(1+o(1))
$$

where $D_{\max }=D / d$. On the other hand, using the Poisson point process probabilities when $d<1$,

$$
P_{\text {out }, 1}=N_{0}\left(D^{-2 / \nu}-D_{\max }^{-2 / \nu}\right)
$$

Comparing (34) and (35), when $d>1$ and $D \rightarrow \infty, P_{\text {out }}=$ $P_{\text {out }, 1} \cdot(1+o(1))$ from which Corollary 1 follows.

\section{Proof of Theorem 2}

The proof is essentially based on Lemma 1 and properties of Poisson point processes. An important property is that the number of nodes in the region $S$ is independent with that in any other disjoint region. Based on that, the distances between randomly-selected nodes and the origin are independent of each other, so that the interference powers coming from individual nodes without ordering are i.i.d random variables. The PDF of the interference power $I$ coming from a single node (without ordering) is:

$$
f(I)=\left\{\begin{array}{cl}
\frac{2}{\nu} \frac{I^{-1-2 / \nu}}{R_{\max }^{2}-R_{s}^{2}} & \text { if } R_{\max }^{-\nu} \leq I \leq R_{s}^{-\nu} \\
0 & \text { otherwise }
\end{array}\right.
$$

Let $x_{1}, \cdots, x_{N}$ be the INRs coming from individual nodes without ordering after normalizing by $D_{1}$, so that the PDF of $x=I /\left(P_{0} D_{1}\right)$ is

$$
f(x)=\left\{\begin{array}{cl}
\frac{2}{\nu} \frac{x^{-1-2 / \nu}}{N_{r}} & \text { if } N_{\max }^{-\nu / 2} \leq x \leq N_{s}^{-\nu / 2} \\
0 & \text { otherwise }
\end{array}\right.
$$

where $N_{r}=\pi \lambda\left(R_{\max }^{2}-R_{s}^{2}\right)$ is the average number of nodes in the ring between the circles of radii $R_{\max }$ and $R_{s} ; N_{\max }=$ $\pi \lambda R_{\max }^{2}$ is the average number of nodes in the disk of the radius $R_{\text {max }} ; N_{s}=\pi \lambda R_{s}^{2}$ is the average number of nodes in the disk of the radius $R_{s}$. The MGF of $x$ is

$$
M_{x}(\theta)=\frac{2}{\nu N_{r}} \int_{N_{\max }^{-\nu / 2}}^{N_{s}^{-\nu / 2}} \exp (\theta x) x^{-1-2 / \nu} d x
$$

When $D_{\max }<\infty, 0<N_{s}^{-\nu / 2}<\infty$. Since $0<N_{\max }^{-\nu / 2}<\infty$, $0<N_{s}^{-\nu / 2}<\infty$, and $0<\exp (\theta x) x^{-1-2 / \nu}<\infty$ 
when $N_{\max }^{-\nu / 2}<x<N_{s}^{-\nu / 2}$ and $\theta<\infty$, it follows that $0<M_{x}(\theta)<\infty$, and

$$
\sup \left\{\theta: M_{x}(\theta)<\infty\right\}=\infty
$$

Since the conditions of Lemma 1 are satisfied, the saddlepoint approximation can now be used.

The saddle point $\hat{\theta}$ can be found by solving the stationarity condition $N_{r} M_{x}^{\prime}(\hat{\theta})=d_{1}$, where $d_{1}=D / D_{1}$ is the normalized threshold INR. After changing the variables, the saddle-point equation becomes

$$
d_{1}=p \int_{a}^{b} \exp (\hat{\theta} x) x^{-p} d x
$$

where $p=2 / \nu<1(\nu>2) ; a=N_{\max }^{-\nu / 2}$ and $b=N_{s}^{-\nu / 2}$. Unfortunately, this is a transcendental equation for which an analytic solution is not known. It can be solved numerically in an efficient way (since the right hand-side is a well-behaved monotonic function of $\hat{\theta}$ ) using any of known techniques. Below, we give an asymptotic analytic solution to obtain some insights unavailable from a purely-numerical solution. When $d_{1} \rightarrow \infty$, it follows that $\hat{\theta} \rightarrow \infty$. Using the integration by parts [19], (40) can be further reduced to

$$
d_{1}=\frac{p \exp (\hat{\theta} b)}{\hat{\theta} b^{p}}[1+o(1)]
$$

as $d_{1} \rightarrow \infty$. Using the standard tools of asymptotic analysis (see e.g. [19][20]), one obtains, after some lengthy but straightforward manipulations, an asymptotic solution for $\hat{\theta}$ (keeping the two leading terms):

$$
\begin{aligned}
\hat{\theta} & =\frac{1}{b} \ln \left(\frac{d_{1} b^{p-1}}{p}\right)+\frac{1}{b} \ln \ln \left(\frac{d_{1} b^{p-1}}{p}\right)(1+o(1)) \\
& =N_{s}^{\nu / 2}(\ln w+\ln \ln w)+o(\ln \ln w)
\end{aligned}
$$

where $w=\nu d_{1} N_{s}^{\nu / 2-1} / 2$.

Let us now evaluate $z_{2}$ and $z_{3}$. From Lemma $1, z_{2}$ can be expressed as:

$$
z_{2}=\operatorname{sgn}(\hat{\theta})\left(2 \hat{\theta} d_{1}-2 N_{r}\left(M_{x}(\hat{\theta})-1\right)\right)^{1 / 2}
$$

where

$$
\begin{aligned}
N_{r}\left(M_{x}(\hat{\theta})-1\right) & =p \int_{a}^{b} \exp (\hat{\theta} x) x^{-1-p} d x-N_{r} \\
& =\left(d_{1} N_{s}^{\nu / 2}+N_{s}\right)(1+o(1))
\end{aligned}
$$

so (14) follows. From Lemma $1, z_{3}$ can now be expressed as:

$$
\begin{aligned}
z_{3} & =\hat{\theta} \sqrt{N_{r} M_{x}^{\prime \prime}(\hat{\theta})}=\hat{\theta} \sqrt{p \int_{a}^{b} \exp (\hat{\theta} x) x^{1-p} d x} \\
& =\hat{\theta} \sqrt{d_{1} N_{s}^{-\nu / 2}}(1+o(1))
\end{aligned}
$$

and from Lemma 1, an asymptotic solution for $\rho_{c 4}(\theta)$ can be expressed as

$$
\begin{aligned}
\rho_{c 4}(\theta) & =\frac{\nu}{2} \frac{\int_{N_{\max }^{-\nu / 2}}^{N_{-\nu / 2}^{-\nu}} \exp (\theta x) x^{3-2 / \nu} d x}{\left[\int_{N_{\max }^{-\nu / 2}}^{N_{s}^{-\nu / 2}} \exp (\theta x) x^{1-2 / \nu} d x\right]^{2}} \\
& =\frac{\nu b}{2} \frac{\theta b^{2 / \nu}}{\exp (\theta b)}[1+o(1)]
\end{aligned}
$$

and using (41)

$$
\rho_{c 4}(\hat{\theta})=b d_{1}^{-1}[1+o(1)]
$$

Finally, using (47) in (8), one obtains (9).

\section{Proof of Corollary 3}

Let us consider the integral $\int_{a}^{b} \exp (\hat{\theta} x) x^{-p} d x$ in (40). It can be upper bounded as

$$
\begin{aligned}
\frac{d_{1}}{p} & =\int_{a}^{b} \exp (\hat{\theta} x) x^{-p} d x \leq a^{-p} \int_{a}^{b} \exp (\hat{\theta} x) d x \\
& \leq \frac{\exp (\hat{\theta} b)}{a^{p} \hat{\theta}}[1-\exp (-\hat{\theta}(b-a))]
\end{aligned}
$$

similarly,

$$
\frac{d_{1}}{p} \geq \frac{\exp (\hat{\theta} b)}{b^{p} \hat{\theta}}[1-\exp (-\hat{\theta}(b-a))]
$$

Using the standard tools of asymptotic analysis (see e.g. [19]), when $x$ is large, the lower bound for $x$ in the equation $e^{x} / x=$ $\mu$ is $x \geq \ln \mu$, so that the lower bound for $\hat{\theta}$ is

$$
\hat{\theta} \geq a_{1} \ln d_{1}
$$

where

$a_{1}=(p \ln a-\ln b-\ln p) / b=\frac{1}{2} N_{s}^{\nu / 2}\left[\nu \ln N_{s}-2 \ln \left(2 N_{\max } / \nu\right)\right]$

When $D \rightarrow \infty$, then $\hat{\theta} \rightarrow \infty$, which implies that $\hat{\theta}>0$ for sufficiently large $D$, so that

$z_{2}^{2} \leq 2 \hat{\theta} d_{1}+2 N_{r}$,

$z_{3}^{2} \geq p a^{1-p} \hat{\theta} \exp (\hat{\theta} b)[1-\exp (-\hat{\theta}(b-a))]=\hat{\theta}^{2} d_{1}(1+o(1))$

and also $z_{3} \geq z_{2}>0$. Therefore, $z_{2}^{-1} \ln \left(z_{3} / z_{2}\right) \geq 0$ and

$$
z_{1}=z_{2}+z_{2}^{-1} \ln \left(z_{3} / z_{2}\right) \geq z_{2} .
$$

On the other hand, when $\hat{\theta}>0$,

$$
z_{2}^{2} \geq 2 \hat{\theta} d_{1}+2 N_{r}-\frac{2 p \exp (\hat{\theta} b)}{a^{1+p} \hat{\theta}}[1-\exp (-\hat{\theta}(b-a))]
$$

and using (49) and (51), one obtain

$$
z_{2}^{2} \geq 2 \hat{\theta} d_{1}+2 N_{r}-\frac{2 d_{1} b^{p}}{a^{1+p}}
$$

Using these facts, the outage probability in (9) can now be bounded as

$$
\begin{aligned}
P_{\text {out }} & =Q\left(z_{1}\right)(1+o(1)) \leq \frac{1}{2} \exp \left(-z_{1}^{2} / 2\right)(1+o(1)) \\
& \leq \frac{1}{2} \exp \left(-z_{2}^{2} / 2\right)(1+o(1)) \\
& \leq \frac{1}{2} \exp \left\{-d_{1}\left(a_{1} \ln d_{1}-\frac{N_{\max }^{1+2 / \nu}}{N_{s}}+\frac{N_{r}}{d_{1}}\right)\right\}(1+o(1))
\end{aligned}
$$




\section{E. Proof of Theorem 3}

From the system model, the SUs are randomly located according to a Poisson point process, so that the CDF of the distance $r_{k}$ between $k_{t h}$ nearest node and the origin is

$$
\operatorname{Pr}\left\{r_{k}<R_{D}\right\}=F_{k}\left(\pi \lambda\left(R_{D}^{2}-R_{s}^{2}\right)\right)
$$

if $R_{D}>R_{s}$ and 0 otherwise, where

$$
F_{k}(x)=1-\sum_{i=0}^{k-1} \frac{x^{i}}{i !} \exp (-x)
$$

Let $d=D / D_{\max }<1$. After canceling interference from $(k-1)$ nearest nodes, the probability of the $k_{t h}$ nearest node along causing an outage is

$$
\begin{aligned}
P_{\text {out }, k} & =\operatorname{Pr}\left\{I_{k}>D P_{0}\right\}=\operatorname{Pr}\left\{r_{k}<R_{D}\right\} \\
& =F_{k}\left(\frac{N_{0}}{D^{2 / \nu}}\left(1-d^{2 / \nu}\right)\right)
\end{aligned}
$$

where $I_{1} \geq I_{2} \geq \ldots \geq I_{N}$ are ordered interference powers. When $D \rightarrow \infty$, (55) simplifies to

$$
P_{\text {out }, k}=\frac{1}{k !}\left(\frac{N_{0}}{D^{2 / \nu}}\left(1-d^{2 / \nu}\right)\right)^{k}(1+o(1))
$$

We further need the following technical lemma, which is a generalization of Lemma 4.4.2 in [21].

Lemma 2: Let $X$ be a positive random variable with a regularly varying tail, i.e. there is a constant $b>0$ such that $\forall a>1$,

$$
\lim _{x \rightarrow \infty} \frac{\operatorname{Pr}\{X>a \cdot x\}}{\operatorname{Pr}\{X>x\}}=a^{-b}
$$

and let the tail of $X$ to dominate the tail of another positive random variables $Y_{1}$, i.e.

$$
\lim _{x \rightarrow \infty} \frac{\operatorname{Pr}\left\{Y_{1}>x\right\}}{\operatorname{Pr}\{X>x\}}=0
$$

Then

$$
\lim _{x \rightarrow \infty} \frac{\operatorname{Pr}\left\{X+\sum_{i=1}^{n} Y_{i}>x\right\}}{\operatorname{Pr}\{X>x\}}=1
$$

where $Y_{1} \geq Y_{2} \geq \cdots \geq Y_{n}$ are ordered positive random variables and $n<\infty$.

Proof of Lemma 2: It follows from Lemma 4.4.2 in [21] applied to $X$ and $Y_{1}$ that

$$
\lim _{x \rightarrow \infty} \frac{\operatorname{Pr}\left\{X+Y_{1}>x\right\}}{\operatorname{Pr}\{X>x\}}=1
$$

Using (60), it is straight forward to verify that

$$
\lim _{x \rightarrow \infty} \frac{\operatorname{Pr}\left\{X+Y_{1}>x\right\}}{\operatorname{Pr}\{X>x\}}=\lim _{x \rightarrow \infty} \frac{\operatorname{Pr}\left\{X+n \cdot Y_{1}>x\right\}}{\operatorname{Pr}\{X>x\}}=1
$$

Consider now the following bounds

$$
\begin{aligned}
\operatorname{Pr}\left\{X+Y_{1}>x\right\} & \leq \operatorname{Pr}\left\{X+\sum_{i=1}^{n} Y_{i}>x\right\} \\
& \leq \operatorname{Pr}\left\{X+n Y_{1}>x\right\}
\end{aligned}
$$

and use (61) to obtain (59).
Using (55), it is straightforward to verify that

$$
\lim _{x \rightarrow \infty} \frac{\operatorname{Pr}\left\{I_{k+1}>x\right\}}{\operatorname{Pr}\left\{I_{k}>x\right\}}=0
$$

Now, observe that $N$ is finite with probability one and use Lemma 2 with $X=I_{k}, Y_{i}=I_{k+i}, i=1 \ldots N-k$, so that

$$
\lim _{x \rightarrow \infty} \frac{\operatorname{Pr}\left\{\sum_{i=k}^{N} I_{i}>x\right\}}{\operatorname{Pr}\left\{I_{k}>x\right\}}=1
$$

Thus, the outage probability is dominated by the nearest active (k-th) node and 1st equality in (21) follows. 2nd and 3rd equalities follow from (56).

\section{F. Proof of Theorem 4}

The nearest-node INR under fading is $d_{s}=g_{1} I_{1} / P_{0}$. The probability of it exceeding the threshold $D$ (and thus causing an outage event) is given by (64), where $f(g, D)=$ $f_{g}(g) \bar{F}(D / g), \bar{F}(x)=\operatorname{Pr}\left\{I_{1}>D P_{0}\right\}$ is the CCDF of the nearest-node non-fading INR $I_{1} / P_{0}$, and $0<\varepsilon<1$ is a constant. When $g \in\left[0, D / D_{\max }\right), D / g>D_{\max }$, so that $\bar{F}(D / g)=0$ and thus $J_{1}=0$. When $D \rightarrow \infty$,

$$
\begin{aligned}
J_{2} & =\int_{D / D_{\max }}^{\left(D / D_{1}\right)^{\varepsilon}}\left[1-\exp \left(\frac{N_{0}}{D_{\max }^{2 / \nu}}-\frac{N_{0}}{(D / g)^{2 / \nu}}\right)\right] f_{g}(g) d g \\
& =\int_{d}^{\left(D / D_{1}\right)^{\varepsilon}}\left[1-\exp \left(-\frac{N_{0}}{D^{2 / \nu}}\left(g^{2 / \nu}-d^{2 / \nu}\right)\right)\right] f_{g}(g) d g \\
& =N_{0} D^{-2 / \nu} \int_{d}^{\left(D / D_{1}\right)^{\varepsilon}}\left(g^{2 / \nu}-d^{2 / \nu}\right)(1+o(1)) f_{g}(g) d g \\
& =N_{0} D^{-2 / \nu} \int_{d}^{\infty}\left(g^{2 / \nu}-d^{2 / \nu}\right) f_{g}(g) d g \cdot(1+o(1)) \\
& =N_{0} D^{-2 / \nu} M_{1}(d) \cdot(1+o(1))
\end{aligned}
$$

where $d=D / D_{\max }$. On the other hand,

$$
\begin{aligned}
J_{3} & =\int_{\left(D / D_{1}\right)^{\varepsilon}}^{\infty} f_{g}(g) \bar{F}(D / g) d g \\
& \leq \int_{\left(D / D_{1}\right)^{\varepsilon}}^{\infty} f_{g}(g) d g=1-F_{g}\left(\left(D / D_{1}\right)^{\varepsilon}\right)=o\left(J_{2}\right)
\end{aligned}
$$

since $1-F_{g}(x)$ decays faster than polynomially, and thus $J_{3}$ can be neglected, so that

$$
P_{\text {out }, 1}=J_{2}(1+o(1))=N_{0} D^{-2 / \nu} M_{1}(d) \cdot(1+o(1))
$$

In a similar way, using (55), one obtains $P_{\text {out }, k}$ :

$$
P_{\text {out }, k}=\operatorname{Pr}\left\{g_{k} I_{k}>D P_{0}\right\}=\frac{M_{k}(d)}{k !}\left(\frac{N_{0}}{D^{2 / \nu}}\right)^{k}(1+o(1))
$$

Observe further that $P_{\text {out }, k}$ has regularly-varying tails and that the tail of $g_{1} I_{1}$ dominates that of $g_{2} I_{2}$, so that Lemma 2 applies and thus (23) follows.

\section{G. Proof of Proposition 4}

The outage probability caused by the $k$-th nearest node alone under fading is given by (68). Using Lemma 2 and the reasoning similar to that in the proofs of Theorems 3 and 4, (24) follows, i.e. the nearest active node dominates the outage probability for a broad class of fading distributions whose tails decay faster than polynomially. 


$$
P_{\text {out }, 1}=\operatorname{Pr}\left\{d_{s}>D\right\}=\int_{0}^{\infty} f(g, D) d g=\underbrace{\int_{0}^{D / D_{\max }} f(g, D) d g}_{J_{1}}+\underbrace{\int_{D / D_{\max }}^{\left(D / D_{1}\right)^{\varepsilon}} f(g, D) d g}_{J_{2}}+\underbrace{\int_{\left(D / D_{1}\right)^{\varepsilon}}^{\infty} f(g, D) d g}_{J_{3}}
$$

\section{REFERENCES}

[1] Special Issue: “Cognitive Radio Part 2: Fundamental Issues,” Proc. IEEE, vol. 97, no. 5, May. 2009.

[2] M. Haenggi and R. K. Ganti, "Interference in Large Wireless Networks," Foundations and Trends in Networking, vol. 3, no. 2, pp. 127 - 248, 2009.

[3] E. S. Sousa and J. A. Silvester, "Optimum Transmission Tanges in a Direct-Sequence Spread-Spectrum Multihop Packet Radio Network," IEEE J. Sel. Areas Commun., vol. 8, no. 5, pp. 762 - 771, Jun. 1990.

[4] J. Ilow and D. Hatzinakos, "Analytic Alpha-Stable Noise Modeling in a Poisson Field of Interferers or Scatterers," IEEE Trans. Signal Process., vol. 46, no. 6, pp. 1601 - 1611, Jun. 1998.

[5] S. Weber, J. Andrews, X. Yang, and G. de Veciana, "Transmission Capacity of Wireless Ad Hoc Networks With Successive Interference Cancellation,” IEEE Trans. Inf. Theory, vol. 53, pp. 2799 - 2814, Aug. 2007.

[6] V. Mordachev and S. Loyka, "On Node Density - Outage Probability Tradeoff in Wireless Networks," IEEE J. Sel. Areas Commun., vol. 27, no. 7, pp. 1120 - 1131, Sept. 2009.

[7] O. B. S. Ali, C. Cardinal and F. Gagnon, "On the Performance of Interference Cancellation in Wireless Ad Hoc Networks," IEEE Trans. Commun., vol. 58, no. 2, pp. 433 - 437, Feb. 2010.

[8] M. Aljuaid and H. Yanikomeroglu, "Investigating the Gaussian Convergence of the Distribution of the Aggregate Interference Power in Large Wireless Networks," IEEE Trans. Vech. Technol., vol. 59, no. 9, pp. 4418 - 4424, Nov. 2010.

[9] A. Ghasemi and E. S. Sousa, "Interference Aggregation in SpectrumSensing Cognitive Wireless Networks," IEEE J. Sel. Topics Signal Process., vol. 2, no. 1, pp. 41 - 56, Feb. 2008.

[10] X. Hong, C. X. Wang and J. Thompson, "Interference Modeling of Cognitive Radio Networks", IEEE VTC Spring 2008, Singapore, May. 2008.

[11] A. Rabbachin, T. Q. S. Quek, H. Shin and M. Z. Win, "Cognitive Network Interference ," IEEE J. Sel. Areas Commun., vol. 29, no. 2, pp. 480 - 493, Jan. 2011.

[12] A. J. Ganesh and G. L.Torrisi, "Large Deviations of the Interference in a Wireless Communication Model," IEEE Trans. Inf. Theory, vol. 54, no. 8, pp.3505 - 3517, Aug. 2008.

[13] J. L. Jensen, Saddlepoint Approximation. Oxford University Press, Oxford, 1995.

[14] Y. Wen, S. Loyka and A. Yongacoglu, "The Impact of Fading on the Outage Probability in Cognitive Radio Networks," IEEE VTC 2010 Fall, Sept. 2010.

[15] Y. Wen, S. Loyka and A. Yongacoglu, "On Distribution of Aggregate Interference in Cognitive Radio Networks," 25th Biennial Symposium on Communications (QBSC - 10), May. 2010.

[16] R. N. Mantegna and H. E. Stanley, "Stochastic Process with Ultraslow Convergence to a Gaussian: the Truncated Levy Flight," Physical Review Letters, vol. 73, no. 22, pp. 2946 - 2949, Nov. 1994.

[17] O. B. Nielsen and D. Cox, Asymptotic Techniques for Use in Statistics. New York: Chapman and Hall, 1989.

[18] Y. Wen, "Outage Probability and Agregate Interference in Cognitive Radio Networks," PhD Thesis, University of Ottawa, 2012.

[19] F. W. J. Olver, Asymptotics and Special Functions. New York: Academic Press, 1997.

[20] M. A. Efgrafov, Asymptotic Expansions and Entire Functions. Moscow: GITTL, 1957.

[21] G. Samorodnitsky and M. S. Taqqu, Stable Non-Gaussian Random Processes. New York: Chapman and Hall, 1994.
[22] G. A. Korn and T. M. Korn, Mathematical Handbook for Scientists and Engineers. Dover, New York, 2000.

[23] D. Niyato and E. Hossain, "Spectrum Trading in Cognitive Radio Networks: A Market-Equilibrium-Based Approach," IEEE Wireless Commun. Mag., vol.15, pp.71-80, Dec. 2008.

[24] H. L. Van Trees, Optimum Array Processing, Wiley, New York, 2002.

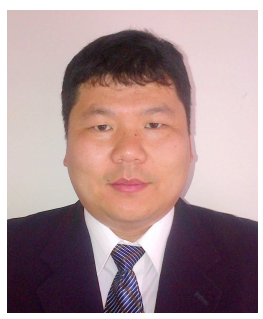

Yaobin Wen received the B.S. degree in Electrical Engineering from Nanjing University, China, in 1996. He received the M.A.Sc. degree in Electrical Engineering from Carleton University, Canada, in 2007. Since 2007, he has been with the School of Electrical Engineering and Computer Science of the University of Ottawa, where he is now a Ph.D. candidate. His research interests are in wireless communication and signal processing.

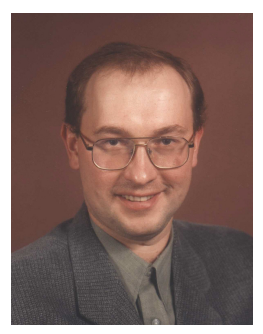

Sergey Loyka was born in Minsk, Belarus. He received the Ph.D. degree in Radio Engineering from the Belorussian State University of Informatics and Radioelectronics (BSUIR), Minsk, Belarus in 1995 and the M.S. degree with honors from Minsk Radioengineering Institute, Minsk, Belarus in 1992. Since 2001 he has been a faculty member at the School of Information Technology and Engineering, University of Ottawa, Canada. Prior to that, he was a research fellow in the Laboratory of Communications and Integrated Microelectronics (LACIME) of Ecole de Technologie Superieure, Montreal, Canada; a senior scientist at the Electromagnetic Compatibility Laboratory of BSUIR, Belarus; an invited scientist at the Laboratory of Electromagnetism and Acoustic (LEMA), Swiss Federal Institute of Technology, Lausanne, Switzerland. His research areas include wireless communications and networks, MIMO systems and smart antennas, RF system modeling and simulation, and electromagnetic compatibility, in which he has published extensively. He received a number of awards from the URSI, the IEEE, the Swiss, Belarus and former USSR governments, and the Soros Foundation.

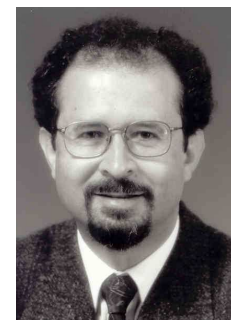

Abbas Yongacoglu received the B.Sc. degree from Bogazici University, Turkey, in 1973, the M.Eng. degree from the University of Toronto, Canada, in 1975, and the Ph.D. degree from the University of Ottawa, Canada, in 1987, all in Electrical Engineering. He worked as a researcher and a system engineer at TUBITAK Marmara Research Institute in Turkey, Philips Research Labs in Holland and Miller Communications Systems in Ottawa. In 1987 he joined the University of Ottawa as an assistant professor. He became an associate professor in 1992, and a full professor in 1996. His area of research is digital communications with emphasis on physical layer of wireless communication systems. 